\title{
EUS-guided gastroenterostomy in management of benign gastric outlet obstruction
}

\section{다 (웅}

\author{
Authors \\ Yen-I Chen ${ }^{1,6}$, Theodore W. James ${ }^{2}$, Amol Agarwal' ${ }^{1}$, Todd H. Baron ${ }^{2}$, Takao Itoi ${ }^{3}$, Rastislav Kunda ${ }^{4}$, Jose Nieto ${ }^{5}$, \\ Majidah Bukhari' ${ }^{1}$, Olaya Brewer Gutierrez ${ }^{1}$, Omid Sanaei ${ }^{1}$, Robert Moran ${ }^{1}$, Lea Fayad ${ }^{1}$, Mouen A. Khashab ${ }^{1}$
}

Institutions

1 Division of Gastroenterology and Hepatology, Johns Hopkins Medical Institutions, Baltimore, Maryland, United States

2 Division of Gastroenterology and Hepatology, University of North Carolina, Chapel Hill, North Carolina, United States

3 Division of Gastroenterology and Hepatology, Tokyo Medical University, Shinjuku-ku, Tokyo, Japan

4 Department of Surgical Gastroenterology, Aarhus University Hospital, Aarhus, Denmark

5 Borland-Groover Clinic, Jacksonville, Florida, United States

6 Division of Gastroenterology and Hepatology, McGill University Health Center, McGill University, Montreal, QC, Canada

submitted 13.9.2017

accepted after revision 14.11.2017

Bibliography

DOI https://doi.org/10.1055/s-0043-123468 |

Endoscopy International Open 2018; 06: E363-368

(c) Georg Thieme Verlag KG Stuttgart · New York

ISSN 2364-3722

Corresponding author

Mouen A. Khashab, MD, Johns Hopkins Hospital, 1800

Orleans Street, Sheikh Zayed Tower, Baltimore, MD 21287

Fax: +1-443-373-1438

mkhasha1@jhmi.edu

\section{ABSTRACT}

Background and study aims Endoscopic ultrasoundguided gastroenterostomy (EUS-GE) in malignant gastric outlet obstruction (GOO) appears to be promising; however, its role in benign GOO is unclear. The aim of this study was to ascertain the clinical efficacy and safety of EUS-GE in benign GOO.

Patients and methods This was an international retrospective series involving 5 tertiary centers. Consecutive patients who underwent EUS-GE between 1/2013-10/2016 for benign GOO were included. The primary endpoint was the rate of clinical success defined as ability to tolerate oral intake without vomiting. Secondary endpoints included technical success and rate of adverse events (AE).

Results Overall, 26 patients (46.2\% female; mean age $57.7 \pm 13.9$ years) underwent EUS-GE for benign GOO due to strictures from chronic pancreatitis $(n=11)$, surgical anastomosis $(n=6)$, peptic ulcer disease $(n=5)$, acute pancreatitis $(n=1)$, superior mesentery artery syndrome $(n=1)$, caustic injury $(n=1)$, and hematoma $(n=1)$. Technical success was achieved in $96.2 \%$. Dilation of the lumen apposing metal stent was performed in $13 / 25$ (52\%) with a mean maximum diameter of $14.6 \pm 1.0 \mathrm{~mm}$. Mean procedure time was $44.6 \pm 26.1 \mathrm{~min}$. Clinical success was observed in $84.0 \%$ with a mean time to oral intake of $1.4 \pm 1.9$ days and a median follow-up of 176.5 (IQR: $47-445.75$ ) days. Rate of unplanned re-intervention was $4.8 \%$. $3 \mathrm{AE}$ were noted including 2 misdeployed stents and 1 gastric leak needing surgical intervention following elective GE stent removal. Conclusions EUS-GE is a promising treatment for benign GOO. Larger and prospective data are needed to further validate this novel endoscopic technique in treating benign GOO of various etiologies.

\section{Introduction}

Gastric outlet obstruction (GOO) can occur from malignant and benign etiologies. Prior to the identification of $\mathrm{H}$. Pylori and the advent of proton pump inhibitors, peptic ulcer disease (PUD) was the most common cause of GOO [1,2]. Since then, however, malignant disease has become the leading etiology of
GOO [3,4]. Nevertheless, GOO from benign etiologies remains an important and difficult to treat pathology that can occur secondary to different disease processes including PUD, acute and chronic pancreatitis, caustic injury from substance ingestion, NSAID-induced stricture, and Crohn's disease $[1,5]$. In terms of management, endoscopic balloon dilation (EBD) has largely replaced surgery as the first line treatment [1]. Al- 
though, clinical success is high with EBD in peptic strictures, it frequently requires multiple endoscopy sessions with repeat dilations, a risk of perforation in 3-7\%, and limited long-term response of $70-80 \%[1,6-10]$. In addition, EBD may be less successful in other diseases such as caustic injury $[11,12]$ and strictures secondary to pancreatitis due to more severe and extensive fibrostenotic changes [5]. Other treatment modalities such as enteral stenting are associated with limited data to support its regular use while surgery is invasive and associated with significant risk for surgical morbidity [1,13-16].

Endoscopic ultrasound guided gastroenterostomy (EUS-GE) with a lumen apposing metal stent (LAMS) is a novel technique described in the management of $\mathrm{GOO}[17,18]$. It entails the insertion of the LAMS from the stomach to the small bowel distal to the obstruction, thereby effectively bypassing the luminal compromise[19]. Small retrospective and prospective series have shown promising results with high clinical success, safety, and low risk for stent obstruction [20-22]. However, the majority of the data on EUS-GE have involved patients with malignant GOO and its efficacy in benign disease is largely undefined. The primary aim of this international multicenter retrospective series is to ascertain the role of EUS-GE in benign GOO by looking at technical feasibility, clinical success, and safety.

\section{Patients and methods}

This is a retrospective multicenter study involving 5 centers ( 3 North America, 1 Europe, and 1 Asia). The institutional review board at each participating center approved this study. All consecutive patients who underwent EUS-GE for benign GOO between January 2014 and October 2016 were included. Patients were identified using center-specific endoscopic or billing databases. Patients with malignant GOO were excluded. Several patients in this study were individually reported in other separate publications but those were not focused on benign disease [ 18 , 23]. Using electronic medical records, the following data were recorded: patient demographics, etiology of GOO, anatomic site of obstruction (categorized as prepyloric/pyloric, duodenal bulb, second part of duodenum, and descending duodenum), prior endoscopic stenting and/or dilation, total procedure time, type/size/number of stent(s) used, technical success, reasons for technical failure, EUS-GE technical approach, location of needle puncture, whether the LAMS was dilated post insertion and caliber of dilation, procedure related adverse events (AEs) with severity graded per the American Society of Gastrointestinal Endoscopy (ASGE) lexicon [24], time to oral intake, type of diet tolerated after EUS-GE, post-procedure length of hospital stay, need for re-intervention, and total duration of follow-up.

\section{Study endpoints}

The primary endpoint was the rate of clinical success defined as ability to tolerate oral intake without vomiting. Diet tolerated was graded as $0=$ no oral diet, $1=$ liquids only, $2=$ soft solids, 3 = almost complete diet, $4=$ full diet [25]. Secondary end points include procedure time, rate of technical success de- fined as adequate positioning and deployment of the stent as determined endoscopically and radiographically, and rate of AEs with the severity graded per the ASGE Lexicon [24].

\section{EUS-GE techniques}

All patients received intravenous antibiotics immediately prior to the procedure. EUS-GE was performed in an endoscopy unit or in an operating room with general anesthesia and endotracheal intubation. Informed consent was obtained from all patients prior to the procedure. All EUS-GE were performed using therapeutic echoendoscopes by expert endoscopists at tertiary care centers with or without trainee involvement. Decision regarding whether or not subsequent stent removal was performed and timing of stent removal were at the discretion of the endoscopist based on patients' clinical evolution. 1 of the following technical approaches was used (decision regarding the approach used was at the discretion of the endoscopist):

\section{Direct gastroenterostomy}

DGE entails direct puncture of a small bowel loop adjacent to the gastric wall using a therapeutic echoendoscope ( $\mathbf{F i g . 1}$ ). To facilitate the puncture, a forward-viewing gastroscope (GIFQ180 or GIF-Q190, Olympus Corporation, Tokyo, Japan) is first inserted and fluid infused into the small bowel distal to the obstruction. Generally, a total of $500 \mathrm{ml}$ of fluid using a combination of saline, methylene blue, and contrast is infused. A 19gauge needle can then be used as a "finder" needle to locate a small bowel loop closest to the stomach with aspiration of bluetinged fluid confirming the correct puncture site. The puncture position may then be confirmed via enterogram. The needle is withdrawn while keeping the endoscope in a stable position. A cautery-assisted LAMS (Axios stent, Boston Scientific Corporation Inc., Marlborough, MA, USA) is then inserted directly across the stomach and into the small bowel followed by stent deployment forming the gastroenterostomy.

\section{Balloon assisted gastroenterostomy}

The balloon assisted gastroenterostomy (BAGE) method has been developed with the hope of improving small bowel access and possibly facilitating stent insertion with the help of a guidewire. A stone retrieval or dilating balloon is inserted over a wire across the obstruction. Following inflation of the balloon with contrast fluid, EUS-guided puncture of the balloon is performed transgastrically with a 19-gauge needle. A guidewire is then advanced deep into the small bowel to guide subsequent insertion of a LAMS.

\section{EUS-guided double-balloon-occluded gastrojejunostomy} bypass

Recently, a specialized double-balloon enteric tube (Tokyo Medical University Type; Create Medic, Yokohama, Japan) has been introduced to facilitate EUS-GE $[21,26]$ but is not yet available outside Japan. The balloon delivery catheter is inserted over a wire across the obstruction. Water with contrast is then used to inflate both balloons to anchor and seal the small bowel at 2 ends. Saline is then infused generously between the 2 balloons. This water insufflation allows for approximation of 

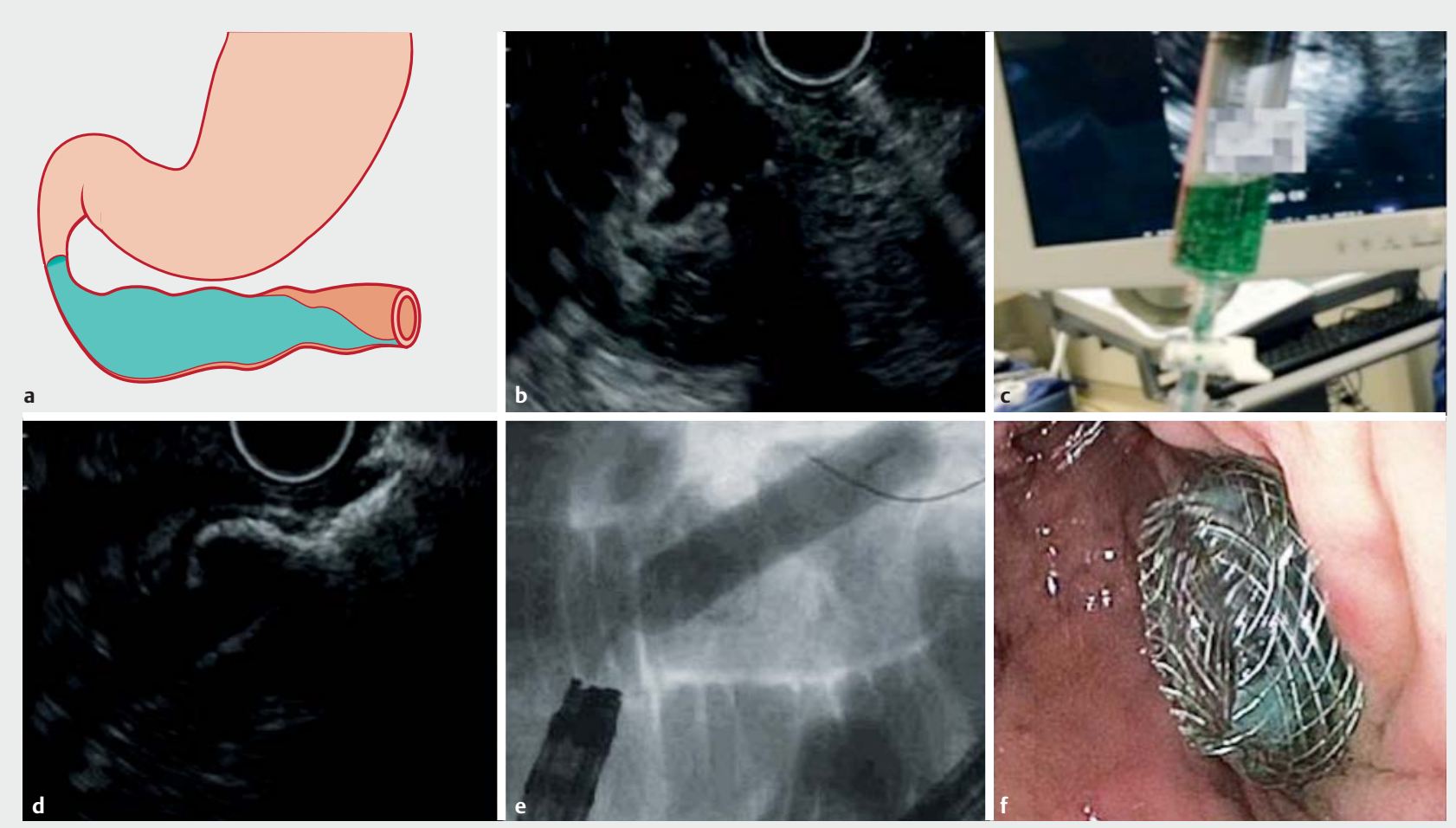

- Fig. 1 Direct EUS-guided gastroenterostomy. a Using a forward-viewing gastroscope, the small bowel is filled with saline mixed with methylene blue and contrast. b Transgastric puncture of the small bowel with a 19-gauge needle. $\mathbf{c}$ Aspiration of blue-tinged fluid confirming the proper location of the puncture. $\mathbf{d}$ LAMS insertion with cautery assistance and stent deployment as seen on EUS. $\mathbf{e}$ Dilation of the stent with a $15-\mathrm{mm}$ radial expansion balloon. $\mathbf{f}$ Endoscopic view of the gastroenterostomy stent post dilation.

small bowel loop to the gastric wall leading to easier and safer transgastric EUS puncture and stent insertion with the cautery tip assisted LAMS.

\section{Statistical analysis}

Continuous variables were reported as means with standard deviations (SD) or, for skewed data, medians with interquartile ranges (IQR). Comparison of linear variables was performed with the t-test and categorical variables by using the chi-square test. A level of significance of $P<0.05$ was adopted for all inferential testing. Statistical analysis was performed by using SPSS version 16 (SPSS Inc, Chicago, III).

\section{Results}

A total of 26 patients (46.2\% female; mean age $57.7 \pm 13.9$ years) underwent EUS-GE for benign GOO due to strictures from chronic pancreatitis ( $n=11,42.3 \%$ ), surgical anastomosis $(n=6,23.1 \%)$, peptic ulcer disease $(n=5,19.2 \%)$, acute pancreatitis $(n=1,3.8 \%)$, superior mesentery artery syndrome due to severe anorexia from colorectal cancer $(n=1,3.8 \%)$, caustic injury ( $n=1,3.8 \%)$, and duodenal wall hematoma from blunt abdominal wall injury $(n=1,3.8 \%)(\triangleright$ Table 1$)$. In the 6 patients with surgical anastomosis the type of surgery and site of obstruction were: Billroth I for gastric cancer with stenosis at the gastoduodenal anastomosis $n=1$, duodenal repair post ERCP perforation complicated by duodenal stricture $n=1$, roux-en-y reconstruction with stricture at the gastrojejunostomy $n=2$, proximal jejunal resection for neuroendocrine tumor complicated by proxima jejunal stricture $n=1$, and undefined $\mathrm{n}=1$. Patients presented with predominant symptoms of nausea and vomiting or abdominal pain in 21 and 5 cases, respectively. The decision to proceed to EUS-GE in patients with conditions such as SMA syndrome, hematoma compression, and acute pancreatitis was due to failure of conservative approach with a feeding tube, severity of patients' symptoms and predicted likelihood of prolonged obstruction. The site of GOO was prepyloric/pyloric in $10(38.5 \%)$ patients, duodenal bulb in 4 (15.4\%), second part of duodenum in 7 (26.9\%), and descending duodenum in 5 (19.2\%). A total of 11 (42.3\%) patients had previous endoscopic therapy including 10 patients who underwent endoscopic balloon dilation (EBD), 3 patients who underwent both EBD and enteral stenting (ES), and 1 patient who underwent ES only. EUS-GE was performed as a first line treatment in 15 (56.7\%) of the patients. Reasons for proceeding directly to EUS-GE include high-grade obstruction with inability to traverse the obstruction with a guidewire to allow for $\operatorname{EBD}(n=1)$ and GOO secondary to etiologies that are unlikely to respond to $\operatorname{EBD}(n=12)$ or at risk of perforation due to ulceration $(n=2)$ near the stricture. The mean number of prior dilation sessions per patient was $2.2 \pm 1.1$ with a mean maximal dilation diameter of $18.5 \pm 2.2 \mathrm{~mm}$. In the 4 patients with previous enteral stenting, stent failure was due to stent migration $(n=1$, esophageal partially covered stent Niti-S TM, Taewoong Medical co, 
- Table 1 Baseline characteristics of patients undergoing EUS-GE for benign $\mathrm{GOO}^{1}$.

\begin{tabular}{|l|c|}
\hline Mean Age \pm SD, years & $57.7 \pm 13.9$ \\
\hline Female $n$ (\%) & $12(46.2)$ \\
\hline Etiology n (\%) & $11(42.3)$ \\
\hline - Chronic pancreatitis stricture & $6(23.1)$ \\
\hline - Surgical anastomosis stricture & $5(19.2)$ \\
\hline - Peptic stricture & $1(3.8)$ \\
\hline Acute pancreatitis & $1(3.8)$ \\
\hline Superior mesenteric syndrome & $1(3.8)$ \\
\hline Caustic stricture & $1(3.8)$ \\
\hline External compression from a hematoma & \\
\hline Location of the obstruction $n=(\%)$ & $10(38.5)$ \\
\hline - Prepyloric/pyloric & $4(15.4)$ \\
\hline - Duodenal bulb & $7(26.9)$ \\
\hline - Second part of the duodenum & $5(19.2)$ \\
\hline - Descending duodenum & $4(15.4)$ \\
\hline History of prior enteral stent $n=(\%)$ & $1(25)$ \\
\hline Mean number of previous enteral stents per patient & $2.3 \pm 1.3$ \\
\hline Reason for enteral stent failure & $10(38.5)$ \\
\hline - Stent obstruction, $n$ (\%) & $2.2 \pm 1.1$ \\
\hline - Stent migration, $\mathrm{n}$ (\%) & $18.5 \pm 2.2$ \\
\hline History of prior dilation & \\
\hline Mean number of previous dilations \pm SD & \\
\hline Mean maximum dilation diameter (mm) \pm SD & \\
\hline Total number of patients=26; SD, standard deviation & \\
\hline
\end{tabular}

South Korea) and stent obstruction ( $n=3$ : LAMS $n=1$, esophageal partially covered stent Niti-S ${ }^{\mathrm{TM}} \mathrm{n}=2$ ).

All EUS-GE procedures were performed with a 15-mm LAMS (cautery-assisted $n=24$, non-cautery-assisted $n=2$ ) with the direct approach ( $n=15,57.7 \%)$, balloon-assisted technique $(n=7$, $26.9 \%)$, or the EPASS technique $(n=4,15.4 \%)$ ( Table 2$)$. Technical success was achieved in 25/26 (96.2\%) cases and dilation of the LAMS performed in 13/25 (52\%) with a mean max diameter of $14.6 \pm 1.0 \mathrm{~mm}$. The mean procedure time was $44.6 \pm 26.1$ minutes.

Clinical success was observed in 21/25 (84.0\%) cases with a median follow-up duration of 176.5 (IQR: 47 -445.75) days. Of those with clinical success, $66.7 \%(14 / 21)$ were able to tolerate a full diet, $14.2 \%(3 / 21)$ an almost a complete diet, $4.8 \%(1 / 21)$ a soft diet, and $14.2 \%(3 / 21)$ a liquid diet. The mean time to per oral intake was $1.4 \pm 1.9$ days. Clinical failure occurred in 4 patients: 2 patients required the insertion of a percutaneous enteric gastrostomy with jejunostomy tube extension (PEG-J) for suspected gastroparesis, 1 patient had PEG insertion for decompression of ileus, and 1 patient was managed surgically.
- Table 2 EUS-GE procedure characteristics ${ }^{1}$.

Type of Procedure

- Direct EUS-GE (\%)

$15(57.7 \%)$

- EPASS

$4(15.4 \%)$

- Balloon Assisted

$7(26.9 \%)$

Procedure time in minutes

$44.6 \pm 26.1$

Part of small bowel punctured

- Distal duodenum

$5(19.2 \%)$

- Jejunum

$20(76.9 \%)$

- Efferent jejunum (surgical anatomy)

$1(3.8 \%)$

Type of Stent Used

\begin{tabular}{|l|c|}
\hline - $15 \mathrm{~mm}$ Cautery-assisted LAMS & $24(92.3 \%)$ \\
\hline - $15 \mathrm{~mm}$ Non-cautery-assisted LAMS & $2(7.7 \%)$ \\
\hline Dilation of LAMS $(\mathrm{n}=25)$ & $13(52 \%)$ \\
\hline Mean maximum dilation diameter $(\mathrm{mm}) \pm$ SD & $14.6 \pm 1.0$ \\
\hline
\end{tabular}

1 Total number of patients=26; EUS-GE, endoscopic ultrasound-guided gastroenterostomy; LAMS, lumen apposing metal stents; EPASS, EUS-guided double-balloon-occluded gastroenterostomy bypass

The latter had failed to respond clinically to the stent and following elective removal of the LAMS at 88 days post EUS-GE was discovered to have gastric leak. The patient underwent emergency laparoscopic gastric wedge resection and Billroth II reconstruction. The patient recovered uneventfully from the surgery and had resolution of the gastric outlet obstruction following resection of the obstructed pylorus secondary to a peptic stricture. The etiology of GOO in patients with clinical failures included peptic stricture $(n=2)$, chronic pancreatitis $(n=1)$, and superior mesenteric artery (SMA) syndrome $(n=1)$ ( $\triangleright$ Fig.2). In terms of safety, there were 2 procedure related AEs, which were rated as mild in severity. These AEs were due to misdeployment of the LAMS with the distal end failing to anchor in the small bowel. Both cases were managed with insertion of a fully covered esophageal stent $18 \times 60 \mathrm{~mm}$ (Niti-S ${ }^{\mathrm{TM}}$, Taewoong Medical co, Korea) through the LAMS to serve as a bridge during the index procedure. No further sequelae were seen in these 2 patients. As aforementioned, there was also a case of gastric leak needing surgical intervention following stent removal. This complication was rated as a severe AE.

In terms of reintervention, of the 21 patients with initial clinical success, unplanned reintervention was performed in only 1 (4.8\%) patient due to stent obstruction secondary to food impaction. This was successfully managed with endoscopic stent cleaning. Overall, 2 patients underwent elective LAMS stent removal with 1 patient due to inadequate clinical response and the other following resolution of GOO due to a large hematoma that subsequently resolved. As aforementioned, the stent removal for the former was complicated by a gastric leak requiring surgery while the latter was uneventful with no observed AE. 


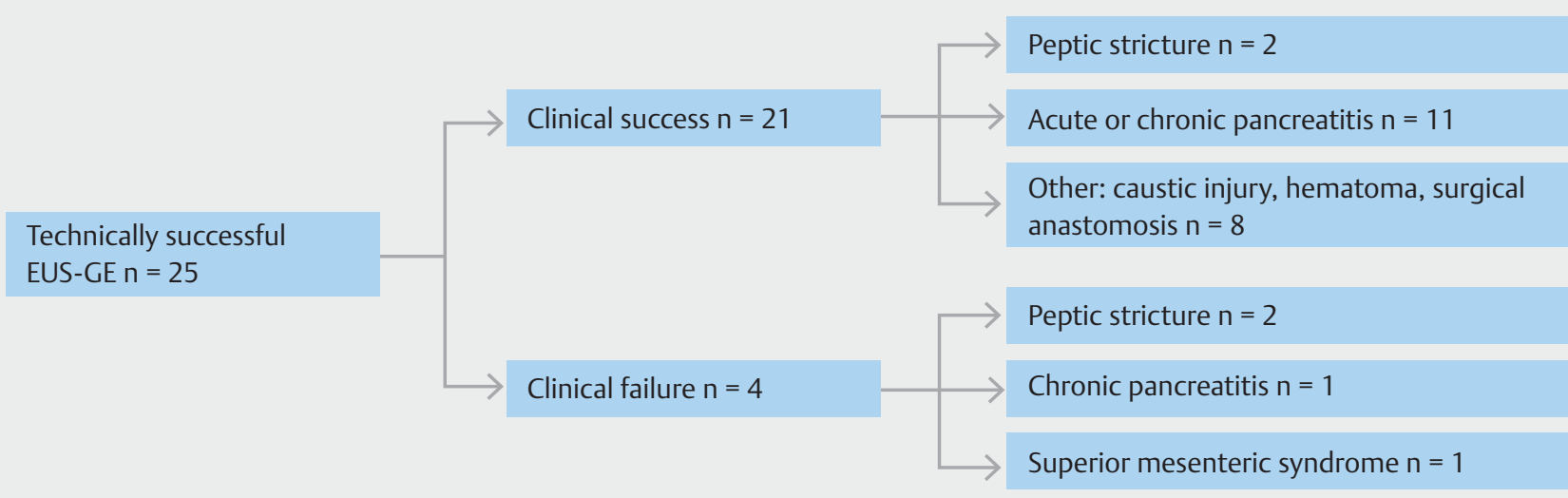

- Fig.2 Clinical outcomes and etiology of gastric outlet obstruction

\section{Discussion}

EUS-GE is a novel and promising modality in the management of GOO. It offers the potential benefits of a surgical bypass while maintaining a minimally invasive approach. Data supporting the use of EUS-GE, however, have largely focused on malignant GOO [20-22]. To our knowledge, this present study is the first published data aiming at ascertaining the role of EUS-GE in benign GOO. Overall, the rates of both technical and clinical success were $96.2 \%$ and $84.0 \%$ respectively, with 3 adverse events noted.

Benign GOO can arise from several etiologies with differing pathophysiology and response to treatment. Endoscopic balloon dilation (EBD) has largely replaced surgery as the initial treatment modality [1]. Clinical success with EBD, however, has been variable in the literature for benign disease. GOO secondary to PUD appears to have the highest response rate to EBD. In fact, $70-80 \%$ of patients can achieve long-term symptomatic relief with the combination of $\mathrm{H}$. pylori eradication and EBD [1,5-10]. However, limited non-surgical options are available in patients who fail EBD. In addition, EBD appears to be have limited effectiveness in other pathologies of benign GOO. In caustic injury induced GOO, for example, EBD requires more endoscopic sessions (range 2 - 13) and appears to be less effective in achieving long-term symptomatic relief $[11,12]$. Strictures associated with chronic pancreatitis can also be especially difficult to treat. In a series of 4 patients treated with EBD for chronic pancreatitis, all 4 patients failed to achieve symptomatic relief and underwent surgical intervention [27]. Poor response to EBD in pancreatitis was also noted in another small series where $50 \%$ of patients needed surgical intervention [28]. Extensive fibrosis and inflammation is believed to be the reason for the refractoriness to dilation. In addition, although generally safe, EBD has been associated with a perforation rate of $3-7 \%[1,7,10]$. Enteral stenting has also been explored in the management of benign GOO. Although small series have shown promising results, stent migration occurs in up to $60 \%$ of the cases with fully covered metal stents, while uncovered metal stents are generally prohibited due to risk of permanent stent anchoring [13-16].

EUS-GE may be a promising modality in benign GOO especially in patients who have failed EBD, those with GOO etiologies that are unlikely to respond to dilation therapy, or when dilation is technically not possible, such as when the obstruction is not traversable with a guidewire. In our series, $84.0 \%$ of the patients achieved clinical success with EUS-GE with $42.3 \%$ of the patients having failed previous EBD and/or ES. Clinical success remained promising at $80.0 \%$ in patients with technically successful EUS-GE and failure with previous EBD and/or ES. The rate of unplanned re-intervention following initial clinical success was also low at $4.7 \%$. This is in contrast to EBD, which often requires several endoscopic sessions to achieve the appropriate luminal diameter especially in non-peptic ulcer induced strictures [1, 6-10]. In addition, the clinical success of EUS-GE is unlikely to vary according to the etiology given that it is bypassing the anatomic site of obstruction instead of restoring luminal patency through the stricture. In terms of safety, there were 2 mild AEs due to stent misdeployment managed successfully with insertion of a bridging fully covered metal esophageal stents. In addition, there was 1 severe AE following elective stent removal resulting in a gastric leak needing emergency surgical management. To our knowledge, this is first reported case of gastric leak post stent removal for EUS-GE and there are currently no data in the literature looking at the safety of gastroenterostomy stent removal. Although EUS-GE is promising, it is important to keep in mind that it is still a developing technique in the management of benign GOO. The long-term effects of an indwelling LAMS and the safety of its removal are still to be further elucidated, while the technique remains to be perfected. Until further data is available, EUS-GE in benign GOO is best reserved for patients who have failed EBD or if EBD is not possible. It may also be considered in $\mathrm{GOO}$ etiologies that tend to have a poor response to dilation, such as strictures secondary to pancreatitis or caustic injury.

Our study is limited by its retrospective design. Consecutive patients were used to limit selection bias and although it is the largest and only series focused on benign GOO, it remains a 
small study involving 26 patients. Also, follow-up time of 176.5 (IQR: $47-445.75)$ days is relatively short and longer follow-up data will be needed to ascertain the long-term clinical effectiveness and safety of EUS-GE. In addition, all procedures were performed by expert endoscopists at tertiary centers; therefore, our results may not be generalizable to community hospitals. As with any developing, novel technique, EUS-GE should be performed by experienced endoscopists at centers with the appropriate surgical and interventional radiology backup.

\section{Conclusion}

In conclusion, EUS-GE is a promising modality for the management benign GOO caused by a variety of etiologies and may be especially useful in those who fail to respond to endoscopic balloon dilation. Questions regarding the safety of long-term stent indwelling and subsequent stent removal, however, remain to be elucidated. Larger studies and prospective data are needed to further validate this novel endoscopic technique in treating benign GOO.

\section{Competing interests}

Yen-I Chen is a consultant for Bostonc Scientific. Todd H. Baron is a consultant and speaker for Boston Scientific and Olympus. Rastislav Kunda is a consultant, speaker, and medical advisory board for Boston Scientific and Omega medical Imaging. Jose Nieto is a consultant for Boston Scientific. Mouen A. Khashab is a consultant for Boston Scientific and Olympus.

\section{References}

[1] Committee ASoP. Banerjee S, Cash BD et al. The role of endoscopy in the management of patients with peptic ulcer disease. Gastrointest Endosc 2010; 71: 663-668

[2] Paimela H, Tuompo PK, Perakyl T et al. Peptic ulcer surgery during the H2-receptor antagonist era: a population-based epidemiological study of ulcer surgery in Helsinki from 1972 to 1987. Br J Surg 1991; 78: $28-31$

[3] Johnson CD, Ellis H. Gastric outlet obstruction now predicts malignancy. Br J Surg 1990; 77: $1023-1024$

[4] Khullar SK, DiSario JA. Gastric outlet obstruction. Gastrointest Endosc Clin N Am 1996; 6: 585-603

[5] Kochhar R, Kochhar S. Endoscopic balloon dilation for benign gastric outlet obstruction in adults. World J Gastrointest Endosc 2010; 2: 2935

[6] Boylan JJ, Gradzka MI. Long-term results of endoscopic balloon dilatation for gastric outlet obstruction. Dig Dis Sci 1999; 44: 1883-1886

[7] DiSario JA, Fennerty MB, Tietze CC et al. Endoscopic balloon dilation for ulcer-induced gastric outlet obstruction. Am J Gastroenterol 1994; 89: $868-871$

[8] Kozarek RA, Botoman VA, Patterson D]. Long-term follow-up in patients who have undergone balloon dilation for gastric outlet obstruction. Gastrointest Endosc 1990; 36: 558 - 561

[9] Lam YH, Lau JY, Fung TM et al. Endoscopic balloon dilation for benign gastric outlet obstruction with or without Helicobacter pylori infection. Gastrointest Endosc 2004; 60: 229-233
[10] Solt ], Bajor ], Szabo M et al. Long-term results of balloon catheter dilation for benign gastric outlet stenosis. Endoscopy 2003; 35: 490 495

[11] Chiu YC, Liang CM, Tam W et al. The effects of endoscopic-guided balloon dilations in esophageal and gastric strictures caused by corrosive injuries. BMC Gastroenterol 2013; 13: 99

[12] Kochhar R, Dutta U, Sethy PK et al. Endoscopic balloon dilation in caustic-induced chronic gastric outlet obstruction. Gastrointest Endosc 2009; 69: 800 - 805

[13] Binkert CA, Jost R, Steiner A et al. Benign and malignant stenoses of the stomach and duodenum: treatment with self-expanding metallic endoprostheses. Radiology 1996; 199: 335-338

[14] Choi W], Park JJ, Park J et al. Effects of the temporary placement of a self-expandable metallic stent in benign pyloric stenosis. Gut Liver 2013; 7: $417-422$

[15] Dormann AJ, Deppe H, Wigginghaus B. Self-expanding metallic stents for continuous dilatation of benign stenoses in gastrointestinal tract first results of long-term follow-up in interim stent application in pyloric and colonic obstructions. Z Gastroenterol 2001; 39: 957-960

[16] Heo J, Jung MK. Safety and efficacy of a partially covered self-expandable metal stent in benign pyloric obstruction. World J Gastroenterol 2014; 20: 16721 - 16725

[17] Binmoeller KF, Shah JN. Endoscopic ultrasound-guided gastroenterostomy using novel tools designed for transluminal therapy: a porcine study. Endoscopy 2012; 44: 499-503

[18] Khashab MA, Kumbhari V, Grimm IS et al. EUS-guided gastroenterostomy: the first U.S. clinical experience (with video). Gastrointest Endosc 2015; 82: 932 - 938

[19] Chen YI, Khashab MA. Endoscopic approach to gastrointestinal bypass in malignant gastric outlet obstruction. Curr Opin Gastroenterol 2016: doi:10.1097/MOG.0000000000000292

[20] Chen YI, Itoi T, Baron T et al. EUS-guided gastroenterostomy is comparable to enteral stenting in terms of technical feasibility and clinical success with lower rates of re-intervention: An international multicenter comparative study [abstract]. Digestive Disease week 2016. Gastrointest Endosc 2016; 83: AB168

[21] Itoi T, Ishii K, Ikuchi $\mathrm{N}$ et al. Prospective evaluation of endoscopic ultrasonography-guided double-balloon-occluded gastrojejunostomy bypass (EPASS) for malignant gastric outlet obstruction. Gut 2016; 65: $193-195$

[22] Tyberg A, Perez-Miranda M, Sanchez-Ocana R et al. Endoscopic ultrasound-guided gastrojejunostomy with a lumen-apposing metal stent: a multicenter, international experience. Endosc Int Open 2016; 4: E276-E281

[23] Chen YI, Kunda R, Storm AC et al. EUS-guided gastroenterostomy: a multicenter study comparing the direct and balloon-assisted techniques. Gastrointest Endosc 2017: doi:10.1016/j.gie.2017.07.030

[24] Cotton PB, Eisen GM, Aabakken L et al. A lexicon for endoscopic adverse events: report of an ASGE workshop. Gastrointest Endosc 2010; 71: $446-454$

[25] Khashab M, Alawad AS, Shin EJ et al. Enteral stenting versus gastrojejunostomy for palliation of malignant gastric outlet obstruction. Surg Endosc 2013; 27: $2068-2075$

[26] Itoi T, Tsuchiya T, Tonozuka R et al. Novel EUS-guided double-balloonoccluded gastrojejunostomy bypass. Gastrointest Endosc 2016; 83: $461-462$

[27] Kochhar R, Sethy PK, Nagi B et al. Endoscopic balloon dilatation of benign gastric outlet obstruction. J Gastroenterol Hepatol 2004; 19: $418-422$

[28] Rana SS, Bhasin DK, Chandail VS et al. Endoscopic balloon dilatation without fluoroscopy for treating gastric outlet obstruction because of benign etiologies. Surg Endosc 2011; 25: 1579-1584 
CORRECTION

Chen YI, James TW, Agarwal A et al. EUS-guided gastroenterostomy in management of benign gastric outlet obstruction

Endoscopy International Open 2018; 06: E363-E368. DOI: $10.1055 / \mathrm{s}-0043-123468$

In the above mentioned article was middle name of an author missing. Correct is: Theodore W. James 